\title{
Using radiotracer techniques for coastal hydrodynamic model evaluation
}

\author{
C.E. Hughes ${ }^{\text {a,* }}$, P.L. Airey ${ }^{\text {a }}$, E.B. Duran ${ }^{\text {b }}$, B.M. Miller ${ }^{\text {c }}$, \\ E. Sombrito ${ }^{b}$ \\ a Australian Nuclear Science and Technology Organisation, Menai, NSW 2234, Australia \\ b Philippines Nuclear Research Institute, Quezon City, Philippines \\ c Water Research Laboratory, University of NSW, Manly Vale, NSW 2093, Australia
}

Received 22 May 2003; received in revised form 15 December 2003; accepted 29 December 2003

\begin{abstract}
A three-dimensional (3D) water circulation and contaminant transport model of Manila Bay has been developed with the aim of better understanding the formation and movement of harmful algal blooms. Radiotracer techniques were used to evaluate the model by recording the dispersion of a tracer at depths of 2 and $15 \mathrm{~m}$ near the injection point. The selected tracer was ${ }^{99 \mathrm{~m}} \mathrm{Tc}$ eluted from a molybdenum/technetium medical generator. The rationale for the choice of the tracer and the location of the injection is discussed. At $2 \mathrm{~m}$ the transport was dominated by the prevailing winds, and at $15 \mathrm{~m}$ by tidally induced currents. The development of the hydrodynamic model and its experimental evaluation were iterative processes. The experimental study confirmed the need for full 3D modelling of Manila Bay; quantified the impact of the prevailing wind field on contaminant dispersion near the injection point; and allowed the calculation of transverse dispersivity to guide the selection of parameter values used in the overall model.
\end{abstract}

Crown Copyright (C) 2004 Published by Elsevier Ltd. All rights reserved.

Keywords: Radiotracing; ${ }^{99 \mathrm{~m}} \mathrm{Tc}$; Finite element modelling; Hydrodynamics; Manila Bay; RMA

\section{Introduction}

Radiotracer techniques were used to evaluate a three-dimensional (3D) water circulation model within Manila Bay, and thereby contribute to an understanding of the mechanism of the formation and movement of harmful algal blooms (HAB).

\footnotetext{
${ }^{*}$ Corresponding author.

E-mail address: ceh@ansto.gov.au (C.E. Hughes).
}

0265-931X/\$ - see front matter Crown Copyright (C) 2004 Published by Elsevier Ltd. All rights reserved. doi:10.1016/j.jenvrad.2004.03.026 
Van Dolah (2000) reported that marine algal toxins were responsible for 60,000 human intoxications per year globally, with an average mortality rate of $1.5 \%$, and increasing incidence rates. In addition to the health effects, there were major economic losses to communities dependent on sea farming (Correles and Maclean, 1995). Knowledge of the composition and sources of nutrients used by HAB species will facilitate prediction of the occurrence of blooms.

The current investigation contributes to this effort by improving methods for predicting the transport of nutrients and algal blooms throughout Manila Bay from any postulated source. Central to the approach was the development of a 3D numerical model of circulation patterns within the bay (Miller et al., 2001), and the evaluation of the model using radiotracer techniques.

Until recently, tracer techniques have primarily been used as a source of independent information on transport processes within the environment (IAEA, 1983, 1991). The current focus on model evaluation is leading to new opportunities for applications at the limit of current knowledge (Airey et al., 2003).

\section{Manila Bay}

Manila Bay (Fig. 1(a)) is approximately $50 \mathrm{~km}$ by $40 \mathrm{~km}$ with depths varying from $15 \mathrm{~m}$ or less over most of the bay up to $30 \mathrm{~m}$ near the entrance. Circulation within the bay is influenced predominately by oceanic tides, wind-driven currents and fresh water inflows. During the monsoons, the volume of water entering the bay through rivers and streams is considerable and leads to significant stratification. The northeast monsoon occurs from October through to January and the
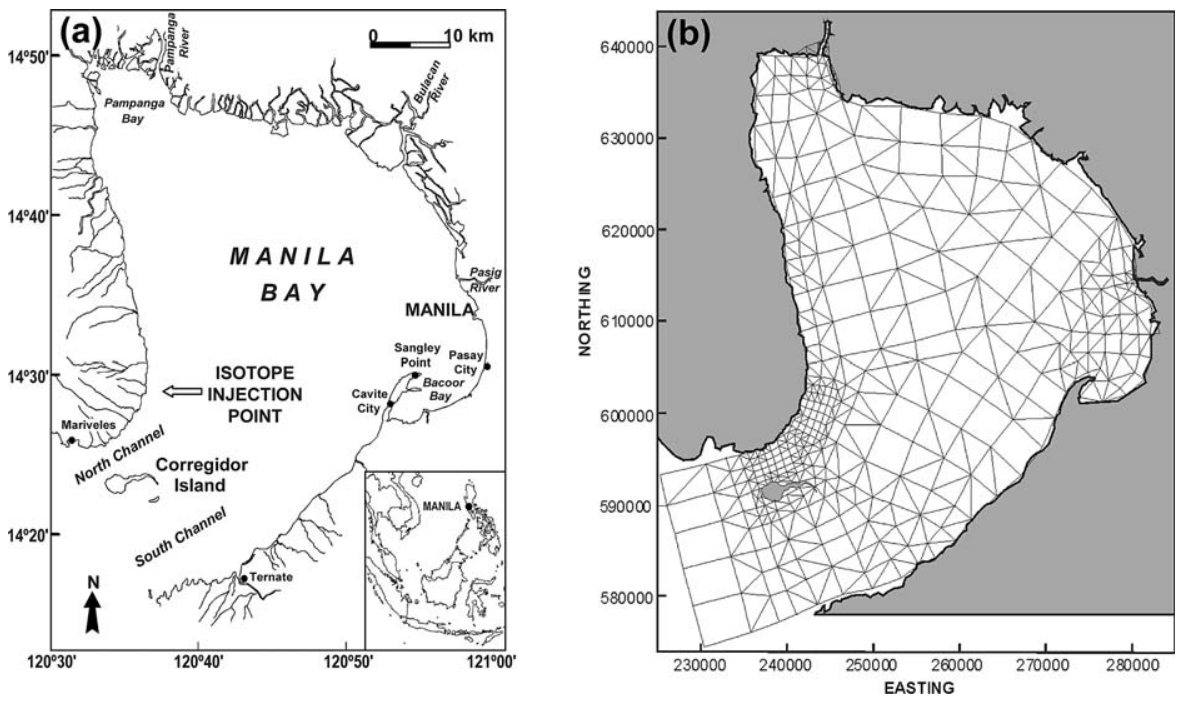

Fig. 1. (a) Manila Bay, Philippines, showing the location of the isotope injection point; (b) finite element mesh. 

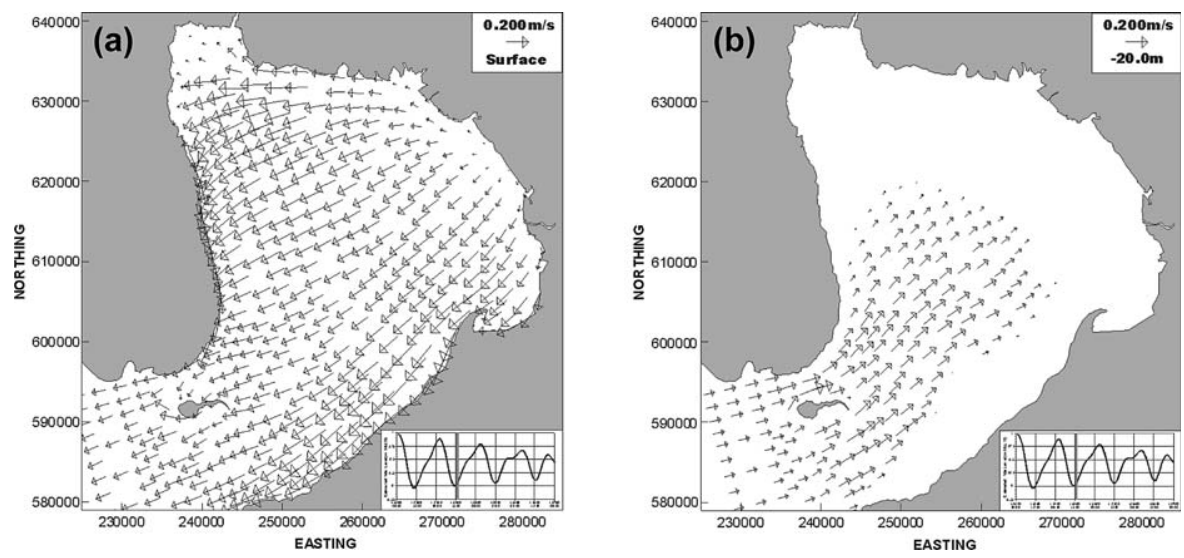

Fig. 2. (a) The surface currents moving with northeast winds, corresponding to the tracer study on 4 December 2000; (b) currents at a depth of $20 \mathrm{~m}$ driven by the tidal fluxes corresponding to the tracer study on 5 December 2000 (Miller et al., 2001).

southwesterly monsoon is from June to September. The tides in Manila Bay are predominately diurnal, with a spring range approximately $2 \mathrm{~m}$ and a neap range of $0.5 \mathrm{~m}$ (Miller et al., 2001).

The predominant winds during the period of the experiment were associated with the northeast monsoon. The hydrodynamic modelling described below generated typical currents at the surface and at depth during this period (Fig. 2). The conditions corresponded to the tracer studies on 4th and 5th December 2000 respectively. Modelled near-surface currents are dominated by wind forcing from the northeast winds, while the currents at $20 \mathrm{~m}$ depth are driven by tidal fluxes leading to vertical stratification and reversal of flows on the incoming tide.

\section{Tracer study}

\subsection{Aim of the Manila Bay study}

The aim of the tracer study was to evaluate the model under two conditions: (a) those reflecting the transport of surface algal blooms; and (b) those reflecting conditions adjacent to bottom sediments that may play a role in initiating the blooms. The approach was to compare the experimental estimates of dispersivity with those used in the model and, if necessary, refine the model to improve the match.

The location of each injection is critical for the success of the investigation as it defines the domain over which direct observations are made. Hence, the tracer was injected at an optimal point for model validation. After consultation with local experts and examination of the hydraulic data, an area on the eastern side of Manila Bay, about $8 \mathrm{~km}$ north of the Corrigedor Island and the north channel at the mouth of the bay was chosen (Fig. 1(a)). 
Each injection was made from the Bureau of Fisheries and Research's oceangoing research vessel. The 30 and 60 min release periods represented a compromise between an instantaneous injection which simplifies data interpretation and the practical need to develop a 'patch' of radiotracer of sufficient size to allow ready monitoring.

\subsection{Choice of tracer}

A wide variety of radiotracers are potentially available. Over 3000 radionuclides are listed in published compilations (Airey and Howard, 2002; Magill, 2000). In practice, about 100 are commonly used. In choosing the radiotracer, a number of questions need to be addressed:

- The integrity of the tracer: Is the key property of the tracer essentially the same as that of the component of interest? In this investigation primary interest lay in the hydrodynamic properties of dissolved or dispersed non-reactive contaminants in Manila Bay.

- The nuclear properties of the tracer: Does the tracer emit a gamma ray with adequate energy and yield for efficient detection? Other factors having been considered, the radiotracer with the shortest half-life is chosen to minimise radiological impact whilst ensuring that detectable life of the tracer is sufficient to match the timescale of the process being studied.

- Radiological and environmental aspects: Does the preparation, handling and ultimate behaviour of the tracer lead to radiological impacts on operators and critical groups within the community that comply with regulations and, in addition, are as low as reasonably achievable (the ALARA principle)? Are there any environmental impacts that are not adequately reflected in human health assessments?

In this instance, the chosen tracer was ${ }^{99 \mathrm{~m}} \mathrm{Tc}$ eluted from a $370 \mathrm{GBq}{ }^{99} \mathrm{Mo} /{ }^{99 \mathrm{~m}} \mathrm{Tc}$ Gentech ${ }^{\circledR}$ medical generator. It emits a $140 \mathrm{keV}$ gamma ray with an efficiency of $89 \%$. Field studies have benefited from the engineering development of medical generators that can be transported and eluted safely and efficiently. The half-life of ${ }^{99 \mathrm{~m}} \mathrm{Tc}(6.0 \mathrm{~h})$ is sufficient to allow an investigation over a full shift. The generators can be used for daily investigations over about a week as the half-life of the parent ${ }^{99} \mathrm{Mo}$ is $2.75 \mathrm{~d}$. A measure of the potential radiological health impact is the dose conversion factor which is the radiation dose absorbed for each unit of activity ingested or inhaled. The dose conversion factor for ${ }^{99 \mathrm{~m}} \mathrm{Tc}$ ingestion of $2.2 \times$ $10^{-11} \mathrm{~Sv} / \mathrm{Bq}$ is small in comparison with other radiotracers.

Chemically, the technetium is eluted from the generator as the pertechnetate ion $\mathrm{TcO}_{4}{ }^{-}$in aerated aqueous saline solution. Under more reducing conditions, and in the absence of complexing agents, the pertechnetate(VII) ion would be reduced to technetium(IV) hydrated oxides (Rard et al., 1999). For the reference species, 
$\mathrm{TcO}(\mathrm{OH})_{2}(\mathrm{aq})$ the relevant electrochemical reaction is

$$
\mathrm{TcO}_{4}^{-}+4 \mathrm{H}^{+}+3 \mathrm{e}^{-} \leftrightarrow \mathrm{TcO}(\mathrm{OH})_{2}(\mathrm{aq})+\mathrm{H}_{2} \mathrm{O}
$$

The calculated standard reduction potential is $0.579 \pm 0.016 \mathrm{~V}$ indicating that the technetium would be predominately in the reduced state in many environmental waters. This value should be used only as a guide, as the chemical processes within Manila Bay would lead to formation of chemical complexes as well as the adsorption of the technetium on finely suspended material. These processes will not compromise the validity of the study, as the settling velocities of the ultrafine particles are very small compared with the advective and dispersive velocities of the water particles with which they are associated. ${ }^{1}$

The short half-life of the technetium, and the rapid dispersion of the tracer combine to ensure that there are no adverse effects on either the local community or the environment.

\subsection{Radiotracer methodology}

The radiotracer injection was as follows: ${ }^{99 \mathrm{~m}} \mathrm{Tc}$ was eluted from one of two 370 GBq generators (calibration date 3 December, 2000) into a shielded vial; transferred from the vial with a shielded syringe into 21 water reservoir; and subsequently metered into Manila Bay at the required depth through Tygon ${ }^{\circledR}$ tubing using a calibrated peristaltic pump.

The tracer surveys were conducted from a smaller Philippine navy inshore patrol vessel. The tracer was monitored using a $5 \mathrm{~cm} \times 5 \mathrm{~cm}$ (2 in.) sodium iodide scintillation detector in a waterproof aluminium housing connected to a Minekin ${ }^{\circledR}$ Model 9302 scalar ratemeter on the monitoring vessel by a cable specified for underwater use.

The detector efficiency was 0.04 counts per second per $\mathrm{Bq} 1^{-1}$ for ${ }^{99 \mathrm{~m}} \mathrm{Tc}$. Detector efficiency was previously determined for similar detectors in 8001 of water in a $1 \mathrm{~m}^{3}$ square tank. This reflects the infinite geometry conditions in Manila Bay as the half thickness for attenuation of the ${ }^{99 \mathrm{~m}} \mathrm{Tc} 140 \mathrm{keV}$ gamma is $4.5 \mathrm{~cm}$.

The areal distribution of the tracer was monitored by towing the detector behind the vessel and vertical distribution by lowering the detector gradually through the water profile. Tracer radioactivity was measured at $2 \mathrm{~s}$ intervals during towing or for $10 \mathrm{~s}$ during depth profiling. Detector depth was not measured directly but was estimated as a function of cable length deployed and the cable angle to the vertical. Consequently, the error in depth is large (estimated at $\pm 20 \%$ ).

Position fixing (GPS) data was recorded and vessel position was tracked through the Ceeman ${ }^{\circledR}$ navigation package. All position co-ordinates referred to in this

\footnotetext{
${ }^{1}$ This assertion has been supported in studies of contaminant dispersion from off shore sewage outfalls at Malabar, North Head (Sydney), Burwood Beach (Newcastle, Australia), and three locations in Hong Kong. In each of these studies either ${ }^{198} \mathrm{Au}$ or ${ }^{99 \mathrm{~m}} \mathrm{Tc}$ was used as a tracer and the results used to evaluate dispersion models. In no cases was there any evidence of settling within a few hours of release.
} 
paper are based on the Clarke 1866 ellipsoid, Luzon datum. Detector position during towing was estimated using a layback algorithm. The output from the radiation detector was combined with the position data in an MS Excel ${ }^{\circledR}$ spreadsheet and visualised using a Surfer ${ }^{\circledR}$ contouring routine. The kriging method of interpolation was used.

\subsection{Manila Bay tracer studies}

Two tracer studies were undertaken on consecutive days at a location about 8 $\mathrm{km}$ north of Corregidor Island and shown in Fig. 1(a).

For the first study, $125 \mathrm{GBq}$ of ${ }^{99 \mathrm{~m}} \mathrm{Tc}$ was injected at a depth of $2 \mathrm{~m}$ over a 30 min period commencing at 12:50 on 4 December 2000. The isotope was released near the bottom of the tide with the wind blowing from the northeast at approximately 13 knots.

The dispersion of the plume was surveyed at a depth of $2 \mathrm{~m}$ between 13:52 and 17:14. The results at four time intervals are shown in Fig. 3. For the purpose of this illustration, the data were grouped into the time intervals listed in the figure.

These conditions were ideal for the study of surface transport. The dominating effect of the wind on the surface transport is apparent from the shape and the translation of the plume. At a depth of $2 \mathrm{~m}$, the plume was moving from the northeast at a velocity of approximately $0.3 \mathrm{~m} \mathrm{~s}^{-1}$. The position of the drogue which was used a guide to the position of the plume is shown in Fig. 3(d). In the absence of the wind field, the effect of the flood tide would be generally in the opposite direction.

Vertical profiles of activity were measured at three locations indicated at positions A and B in Fig. 3(a) and C in Fig. 3(b). The data are plotted in Fig. 4(c). The profiles show significant vertical dispersion over the first $2 \mathrm{~h}$ following commencement of the injection. This observation can be interpreted as resulting from turbulent mixing induced by shear stress between opposing wind and tidally driven current directions as shown in Fig. 2.

The focus of the second investigation on 5th December was the transport of the tracer at depth. For this study $240 \mathrm{GBq}$ of ${ }^{99 \mathrm{~m}} \mathrm{Tc}$ was injected at a depth of $15 \mathrm{~m}$ over a 60 min period commencing at 09:37. The data collected and the relative location of the isotope injection point is shown in Fig. 4(a). The behaviour of the dispersion plume was less well defined than that of the tracer released close to the surface. In contrast to the surface release, there was evidence of significant migration of the tracer north of the injection vessel. This is despite the fact that the prevailing winds were from the northeast.

During this second study, vertical profiles were the primary method of detecting tracer position as it was difficult to determine the most appropriate depth to tow the detector. Selected profiles are marked as AA, BB, CC and DD in Fig. 4(a) and the data are shown in Fig. 4(b). Envelopes of the maximum count rates plotted as individual data points showed considerable variability. There is some evidence for the upward migration of the pulse through the water column during the period of the observation. 

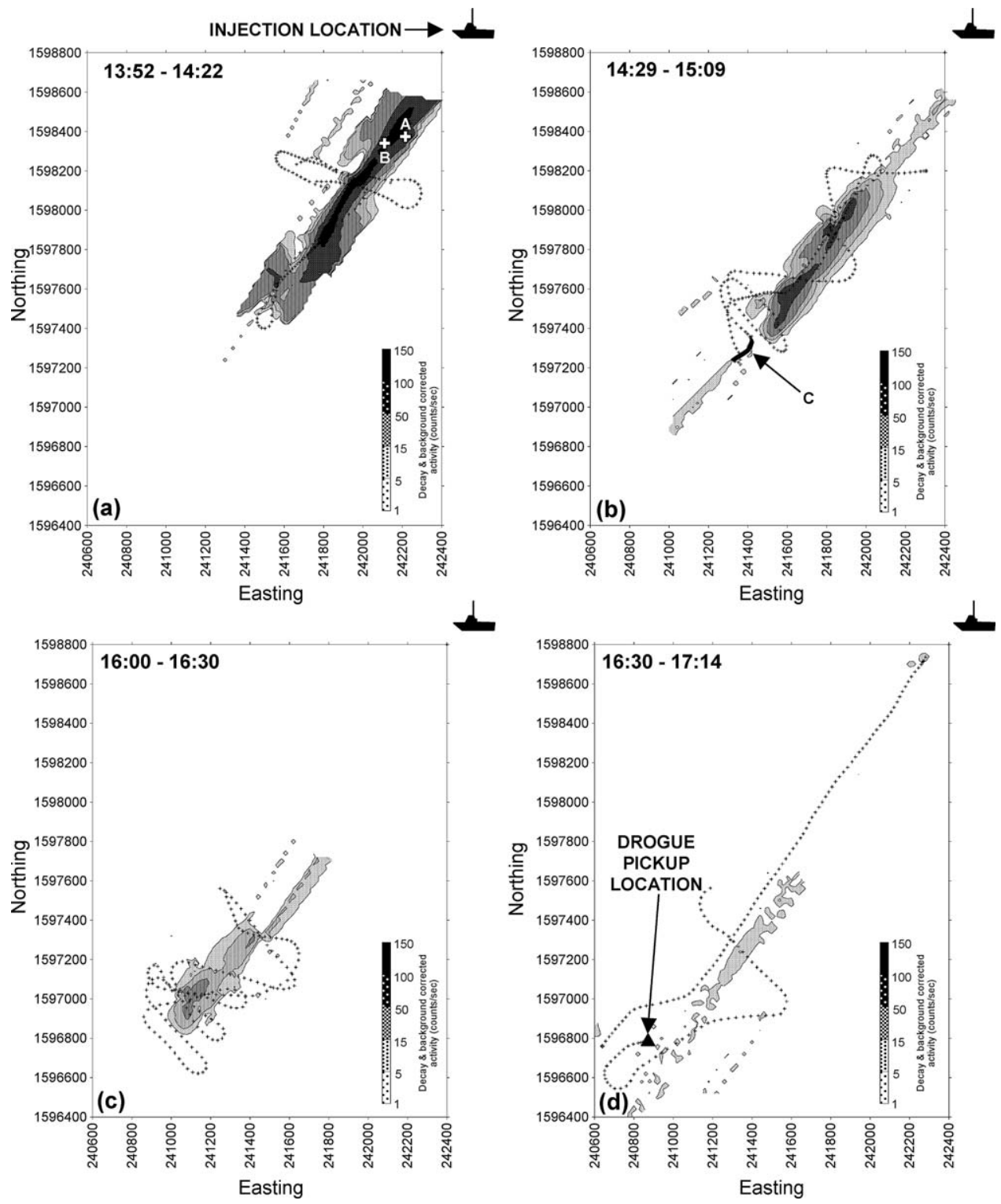

Fig. 3. Areal distribution of tracer released at a depth of $2 \mathrm{~m}$ over a $30 \mathrm{~min}$ period commencing 12:50 on 4 December 2000. Data are grouped into four time intervals (a)-(d) as shown on each figure. The track of the detector, location of the injection vessel and of the drogue at pick-up are shown. Count rate is background and decay corrected to the time of injection. 

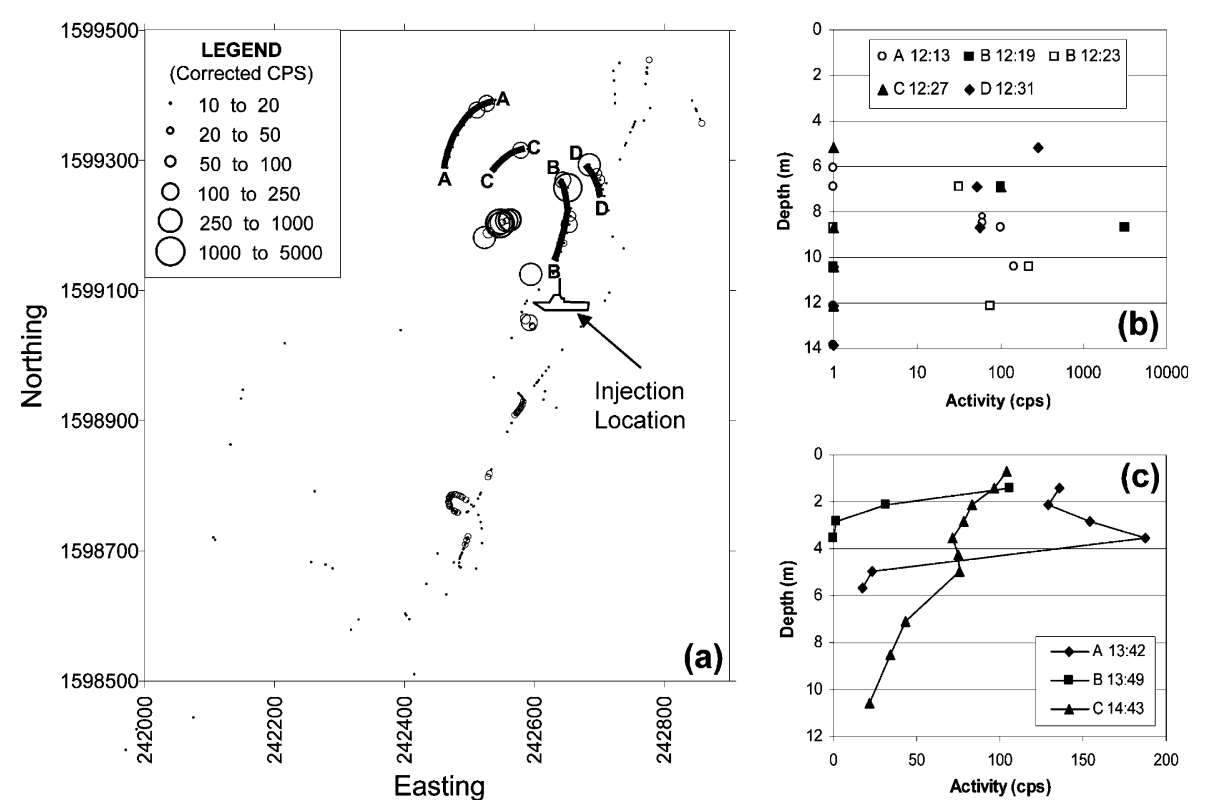

Fig. 4. (a) Areal distribution and (b) vertical profiles of tracer released at a depth of $15 \mathrm{~m}$ over $60 \mathrm{~min}$ commencing 09:37 on 5 December 2000; (c) vertical distribution of tracer from 4 December 2000 at locations A-C shown in Fig. 3. Count rate is background and decay corrected to the time of injection.

\section{Hydrodynamic modelling}

Miller et al. (2001) presents the approach to the hydrodynamic modelling of circulation within Manila Bay in detail; only brief reference will be made here. The 3D finite element model RMA-10 was used (King, 1993). Manila Bay was discretised into a finite element mesh consisting of quadrilateral and triangular elements (Fig. 1(b)). The bathymetry of the mesh was defined using data available from the Admiralty Charts. For 3D hydrodynamic modelling, the model was configured with up to six layers depending on the water depth. Tidal boundary conditions were applied to the outer model boundary.

Wind was applied as a spatially uniform shear over the surface of the model domain varying with time as the wind speed and direction varied. Values of vertical and horizontal eddy diffusivities $\left(0.002\right.$ and $0.5 \mathrm{~m}^{2} \mathrm{~s}^{-1}$, respectively) were selected to best match the movement of the plumes determined by the tracer studies. Four different wind conditions were simulated: (a) no winds; (b) northeasterly winds; (c) southeasterly winds; and (d) southwesterly winds. Apart from case (a) the wind was assumed to be $6 \mathrm{~m} \mathrm{~s}^{-1}$ between 10:00 and 22:00 and $1.5 \mathrm{~m} \mathrm{~s}^{-1}$ at all other times. In this case, the pollutant is released as a pulse at 00:00 on 3 December 2000 at a location about $8 \mathrm{~km}$ north of Corregidor Island, close to the release point of the isotope. 
As there were no current data collected during the experiment, tidal data from Manila Bay, together with estimated values of friction factor and eddy viscosity, were used for the first pass of the model. The parameters were then adjusted to match the tracer data.

The movement of plumes within the predicted hydrodynamic fields were modelled using 3DRWALK (Wang and Miller, 1995). This is a 3D random walk water quality model. For each wind condition, a range of 3DRWALK simulations was undertaken. At each of 13 different locations, a discrete pulse of pollutant was added to the model at a depth of $0.5 \mathrm{~m}$ to start the simulation. The results of these simulations were used to highlight circulation processes within Manila Bay. Animations of these processes provide a basis for assessing various scenarios for the movement of algal blooms around Manila Bay. Further details are presented in Miller et al. (2001).

For demonstration purposes, the model has been set up so that a discrete pulse of pollutant was released in one of 13 locations at a depth of $0.5 \mathrm{~m}$. The net pollutant transport in the absence and presence of the winds over the period from injection until 00:00, 6 December 2000 is visualised in Fig. 5.
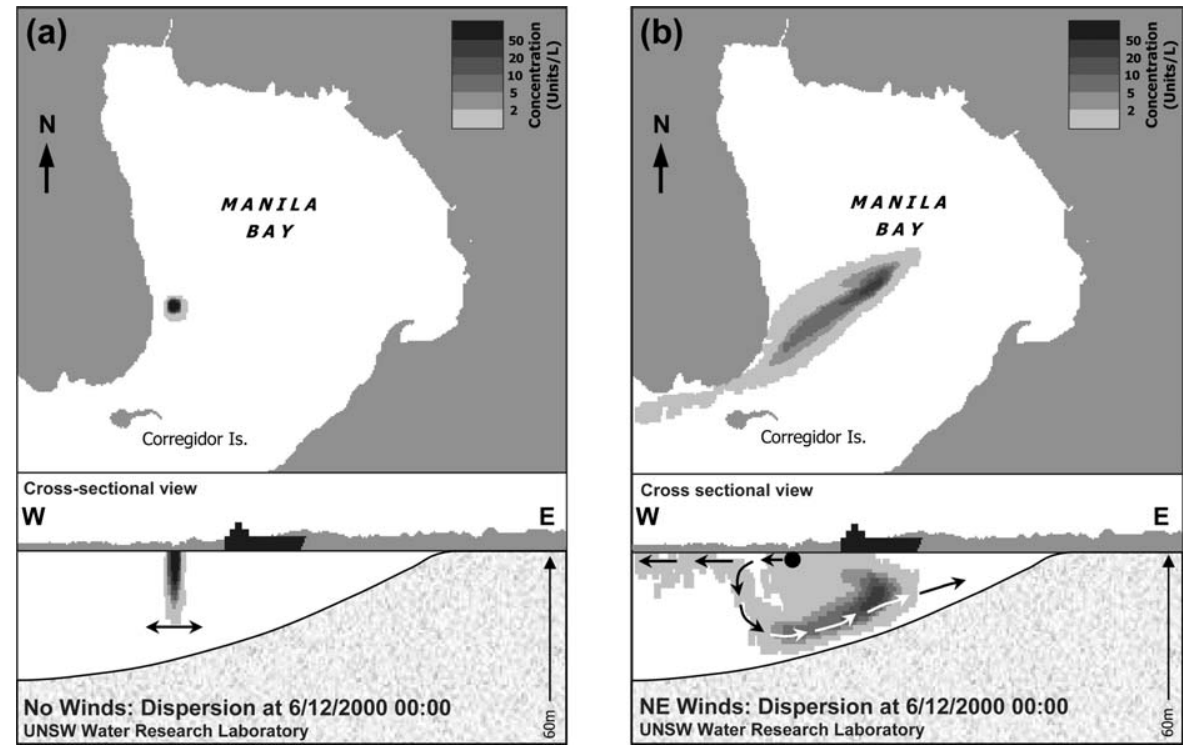

Fig. 5. Illustration of the impact of a northeast wind field on the dispersion of pollutant released as a pulse at 00:00, 3 December 2000, near the isotope injection point, $8 \mathrm{~km}$ north of Corregidor Island. The RMA/3DRWALK codes (King, 1993; Wang and Miller, 1995) were used to model the net effect of the transport in the absence (a) and presence (b) of northeast winds up until 00:00, 6 December 2000. Arrows indicate the prevailing movement of the plume during the simulation. The $\mathrm{E}-\mathrm{W}$ cross sectional views indicate generalised $\mathrm{E}-\mathrm{W}$ vectors of plume movement at its peak concentration in the water column. 


\section{Model evaluation}

The principal aim of the tracer study is to evaluate and refine the hydrodynamic model. The approach is to obtain experimental values of the dispersivities in the general vicinity of the injection point and to use these data to improve the estimates of the parameters used in the bay-wide model. The transverse dispersivity was estimated from the expression:

$$
c=c_{0} \times \mathrm{e}^{-\left[\left(y-y_{0}\right)^{2} / 2 \sigma_{\mathrm{T}}^{2}\right]}
$$

where

$$
\sigma_{\mathrm{T}}(\text { standard deviation for transverse dispersion })=\left(2 \alpha_{\mathrm{T}} x\right)^{1 / 2}
$$

$\left.c(\mathrm{~Bq} \mathrm{~m})^{-3}\right)$ is the tracer concentrations along the transect; $c_{0}\left(\mathrm{~Bq} \mathrm{~m}{ }^{-3}\right)$ is the tracer concentration at the pulse centroid; $\alpha_{\mathrm{T}}(\mathrm{m})\left(=D_{\mathrm{T}} / V\right)$ is the transverse dispersivity; $D_{\mathrm{T}}\left(\mathrm{m}^{2} \mathrm{~s}^{-1}\right)$ is the transverse dispersion coefficient; and $V(=x / T)$ is the mean current velocity in the longitudinal $(x)$ direction.

The distribution of tracer across the plume depicted in Fig. 3(b) is shown in Fig. 6 together with the fitted equation. The parameters in the equation interpreted in terms of Eqs. (2) and (3) yield the following: $2 \sigma_{\mathrm{T}}^{2}=4 \alpha_{\mathrm{T}} \mathrm{x}=1000$. Hence, $\alpha_{\mathrm{T}}=$ $0.149 \mathrm{~m}$ since $x=1679 \mathrm{~m}$. Given that the mean velocity for the pulse is about 0.3 $\mathrm{m} \mathrm{s}^{-1}$, the transverse dispersion coefficient is $0.04 \mathrm{~m}^{2} \mathrm{~s}^{-1}$.

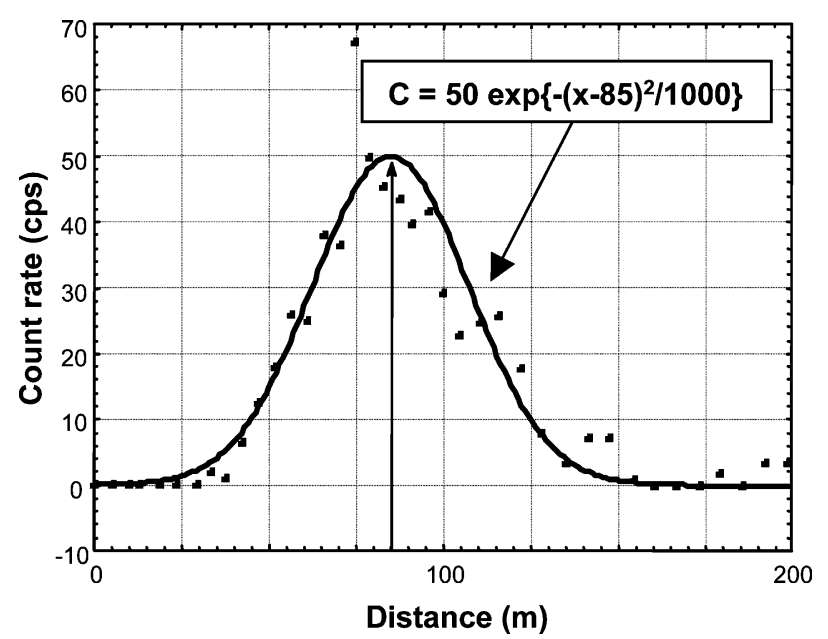

Fig. 6. Variation of the count rate (background and decay corrected) across the transect. The experimental points and an empirical Gaussian equation are shown. The observations were made about 14:45 on 4 December 2002 following an injection between 12:50 and 13:20. The transect is located $1679 \mathrm{~m}$ from the injection point. 


\section{Conclusions}

The following conclusions may be drawn from the study:

1. The tracer study proved extremely useful in evaluating the output of the hydrodynamic model of Manila Bay. The dispersion of the radiotracer released $2 \mathrm{~m}$ from the surface was towards the southwest (Fig. 3) as predicted by the modelled $0.5 \mathrm{~m}$ release (Fig. 5(b)). Again, the transport of the tracer released at depth $(15 \mathrm{~m})$ was in the sector north to northeast as predicted. As this feature would not have been captured in a two-dimensional description of pollutant transport, the data provided experimental justification for the developing the 3D model. An important improvement to the study would have been the concurrent collection of current data using an Acoustic Doppler Current Profiler.

2. The model can be used to evaluate scientific hypotheses for the appearance of algal blooms by allowing a visualisation of the transport of non-reactive solutes or colloids from various potential locations of nutrients within the bay. Such locations could include, for instance, sewage outfalls, sediments and vegetation on the floor of the bay and river inputs. A non-reactive solute circulation model can, of necessity, only contribute to one aspect of the wider problem of predicting the occurrence of algal blooms. For this reason, parallel studies using stable isotope techniques to help in the identification of source of nutrients leading to the growth of algal cysts have been recommended.

3. Considerable effort is being extended in improving and streamlining the techniques used in radiotracing. By taking advantage of modern navigation systems and the capability of integrating data streams from the position fixing systems and monitoring probes, less radiotracer is required for each investigation. Further, it is often possible to use large medical generators as a source of the radiotracer ${ }^{99 \mathrm{~m}} \mathrm{Tc}$, with benefits in terms of safety and convenience. It should be noted that the application of fluorescent tracers is increasing with the development of fluorimeters that can be deployed and provide data in real time. The sensitivities of radiotracers and dye tracers are comparable. However, the latter have limitations in polluted areas where the background fluorescence is high and variable.

4. One of the major technological aims is to progress tracer applications from model evaluation to the greater challenge of model validation. One approach to validation involves reducing the number of independent parameters that may be set by the modeller. A program has begun to use tracers to measure not only dispersivities in three dimensions but also the hydrodynamic shear stress parameter.

\section{Acknowledgements}

The authors wish to acknowledge the major contributions to the project from colleagues in the three organisations. Special gratitude is due to support from the IAEA through the Regional Cooperative Agreement. 


\section{References}

Airey, P., Hughes, C., Kluss, T., Duran, E., Miller, B., Chiuenta, S., Nielsen, L., Hollins, S., 2003. The evolving role of radiotracers in coastal zone studies. Applied Radiation and Isotopes 58, 401-406.

Airey, P.L., Howard, C.J., 2002. Comment on a general property of radionuclides. Journal of Radioanalytical and Nuclear Chemistry 257, 45-46.

Correles, R.A., Maclean, J.L., 1995. Impacts of harmful algae on seafarming in the Asia-Pacific areas. Journal of Applied Phycology 7, 151-162.

IAEA, 1983. Guidebook on Nuclear Techniques in Hydrology. Technical Report Series No. 91, IAEA, Vienna, 439 pp.

IAEA, 1991. Use of Artificial Tracers in Hydrology. IAEA-TECDOC-601, IAEA, Vienna, 230 pp.

King, I., 1993. Sydney deep ocean outfalls environmental monitoring program post commissioning phase: a finite element model for three dimensional density stratified flow. AWACS Interim Report 93/01/04, March 1993.

Magill, J., 2000. Nuclides 2000-An Electronic Chart of the Nuclides, CD ROM, The European Commission, Institute for Transuranium Elements, Karslruhe. Available from http://www.nuclides.net/.

Miller, B.M., Chadwick, M.J., Kluss, T., Hughes, C., Duran, E., 2001. Three dimensional numerical modelling of circulation patterns in Manila Bay, Philippines. In: Proceedings Coasts and Ports 2001, Gold Coast, Qld, The Institution of Engineers Australia, Canberra, pp. 429-434, (ISSN: 1445-9574).

Rard, J.A., Rand, M.H., Andregg, G., Wanner, H., 1999. Chemical Thermodynamics of Technetium, OECD/NEA. North-Holland, Elsevier, Amsterdam, (pp. 74-96).

Van Dolah, F.M., 2000. Marine algal toxins: origins, health effects, and their increased occurrence. Environmental Health Perspective 108 (Suppl. 1), 133-141.

Wang, Y.C., Miller, B.M., 1995. 3DRWALK: A Three Dimensional Lagrangian Particle Transport Model for Pollutant Transport. WRL Technical Report 95/16. 